Applied Physiology, Nutrition, and Metabolism Physiologie appliquée, nutrition et métabolisme

\title{
The dynamics of methionine supply and demand during early development
}

\begin{tabular}{|r|l|}
\hline Journal: & Applied Physiology, Nutrition, and Metabolism \\
\hline Manuscript ID & apnm-2015-0577.R2 \\
\hline Manuscript Type: & Review \\
\hline Date Submitted by the Author: & 22-Feb-2016 \\
\hline Complete List of Authors: & $\begin{array}{l}\text { McBreairty, Laura; Memorial University of Newfoundland, Department of } \\
\text { Biochemistry } \\
\text { Bertolo, Robert; Memorial University of Newfoundland, Department of } \\
\text { Biochemistry }\end{array}$ \\
\hline Keyword: & $\begin{array}{l}\text { neonate, 1-carbon metabolism, methionine requirement, transmethylation, } \\
\text { remethylation }\end{array}$ \\
\hline
\end{tabular}


The dynamics of methionine supply and demand during early development

Laura E. McBreairty, Robert F. Bertolo*

Department of Biochemistry, Memorial University of Newfoundland, St. John's, NL, Canada A1B 3X9

*Corresponding author: Phone: +1 709-864-7954, Fax: +1 709-864-2422, Email:

rbertolo@mun.ca, Address: Department of Biochemistry, Memorial University of

Newfoundland, St. John's, NL, Canada A1B 3X9 


\section{Abstract}

Methionine is an indispensable amino acid which when not incorporated into protein, is converted to the methyl donor S-adenosylmethionine as entry into the methionine cycle. Following transmethylation, homocysteine is either remethylated to reform methionine, or irreversibly transsulfurated to form cysteine. Methionine flux through the methionine cycle and to protein synthesis are high in the neonate and this review focuses on the dynamics of methionine supply and demand during early development when growth requires expansion of pools of protein and transmethylation products such as creatine and phosphatidylcholine (PC). The nutrients folate and betaine (derived from choline) donate a methyl group during remethylation, providing an endogenous supply of methionine to meet methionine demand. During early development, variability in dietary supply of these methionine cycle-related nutrients can affect both the supply and demand of methionine. For example, a greater need for creatine synthesis can limit methionine availability for protein and PC synthesis while increased availability of remethylation nutrients can increase protein synthesis if dietary methionine is limiting. Moreover, changes to methyl group availability early in life can also lead to permanent changes in epigenetic patterns of DNA methylation, which has been implicated in the early origins of adult disease phenomenon. This review aims to summarize how changes in methyl supply and demand can affect availability of methionine for its various functions while highlighting the importance of variability in methionine-related nutrients in the infant diet.

Key words: neonate, 1-carbon metabolism, methionine requirement, transmethylation, remethylation, amino acid 


\section{Introduction}

The indispensable amino acid methionine is primarily partitioned between its two key functions of protein synthesis and transmethylation. Conversion of methionine to Sadenosylmethionine (SAM) represents entry into the methionine cycle (Cantoni 1951) and provides a methyl group for over 50 transmethylation reactions, including creatine and phosphatidylcholine (PC) synthesis, as well as DNA methylation (Schubert et al. 2003) (Fig. 1). During transmethylation, SAM is converted to S-adenosylhomocysteine (SAH) which is further hydrolyzed to homocysteine and adenosine. Homocysteine can undergo the irreversible process of transsulfuration to form cysteine via cystathionine $\beta$-synthase (CBS) (Finkelstein and Chalmers 1970) and cystathionine $\gamma$-lyase (CGL), which has low activity in the neonate (Zlotkin and Anderson 1982). Alternatively, homocysteine can be methylated to reform methionine by accepting a methyl group from either betaine (via betaine-homocysteine methyltransferase (BHMT)) or 5-methyl tetrahydrofolate (via methionine synthase (MS)). So when methionine is not incorporated into protein, it is transmethylated to synthesize a variety of key metabolic products; moreover, this transmethylated methionine can either be recycled through two differently regulated remethylation pathways to regenerate methionine, or undergo irreversible conversion to cysteine. Compared to the adult, the fluxes of methionine to protein synthesis and transmethylation are very high in the growing neonate. Moreover, emerging evidence suggests that variable transmethylation demands and supply of remethylation precursors can dramatically affect methionine availability for protein synthesis. How this variability might affect the current neonatal methionine requirement estimates is unknown.

The total requirement for methionine has been determined in the human neonate to be 38 $\mathrm{mg} \cdot \mathrm{kg}^{-1} \cdot \mathrm{d}^{-1}$, when the conditionally indispensable amino acid cysteine is provided in excess 
(Huang et al. 2012). This review aims to summarize what is known about the dynamics of methionine metabolism in order to highlight how variability of methionine-related nutrients in the infant diet may affect methionine availability and requirements. A considerable amount of research has been conducted in the piglet model to better understand methionine metabolism and requirements during early development. For example, studies in growing piglets have demonstrated that cysteine can spare the methionine requirement by up to $40 \%$ (Shoveller et al. 2003), highlighting the plasticity of the methionine requirement during early development. In piglets, $50 \%$ of methionine is used for transmethylation reactions in the neonate, while the requirement for these reactions is reduced to $30 \%$ of methionine at weaning at 1 month old (Bauchart-Thevret et al. 2009), demonstrating the significant demand of these reactions during early development. Approximately $45 \%$ of homocysteine is remethylated in the neonatal piglet versus $\sim 20 \%$ at 1 month old, demonstrating the significant contribution of remethylation to the total methionine requirement in the neonate (Bauchart-Thevret et al. 2009). Considering cysteine, a product of methionine, can spare the methionine requirement by $40 \%$, it is important to also consider whether variability in the demand for transmethylation reactions, or the supply of remethylation nutrients, can also affect the methionine requirement in the growing neonate. This uncertainty is especially important considering the variability of these nutrients in infant diets.

\section{Transmethylation products}

\section{Creatine}

Synthesis of the energy buffer creatine involves 2 steps, commencing in the kidney with the production of guanidinoacetate (GAA) and ornithine via the transfer of the amidino group from arginine to glycine by arginine:glycine amidinotransferase (AGAT) (Bloch and 
Schoenheimer 1941). This reaction is followed by the SAM-dependent methylation of GAA in the liver via GAMT to produce creatine and SAH (Cantoni and Vignos 1954). Total body creatine in the neonatal piglet increases by $\sim 70 \%$ during the period from 4 to 11 days of age demonstrating the high demand for creatine during early development (Brosnan et al. 2009). The neonatal pig only receives $25 \%$ of its creatine requirement from sow's milk in that period and consequently must synthesize the majority of its creatine needs (Brosnan et al. 2009).

A recent analysis of breast milk and infant formula found a wide variability in the creatine concentration with values up to $334 \mu \mathrm{M}$ in standard infant formula and $\sim 10 \mu \mathrm{M}$ in soybased formula, with human breast milk intermediate at $\sim 70 \mu \mathrm{M}$ (Edison et al. 2013). The contribution between dietary and endogenously synthesized creatine in the human infant is variable with breast-fed infants receiving only $\sim 10 \%$ of their creatine from diet while infants receiving cow milk-based formulas consume up to $\sim 35 \%$ of their total creatine via diet (Edison et al. 2013). Furthermore, infants fed soy-based formula consume negligible amounts of dietary creatine, thus requiring synthesis of the entire creatine requirement. This inconsistency in infant dietary creatine intake translates into variability in the demands for creatine synthesis, which consequently creates a variable demand for methyl groups and methionine. This potentially high methyl demand is important during early development, as neonatal piglet models suggest a high demand for all transmethylation reactions (Bauchart-Thevret et al. 2009).

Dietary creatine supplementation in the rat leads to lower AGAT activity (Walker 1960) as well as lower levels of AGAT mRNA and protein (McGuire et al. 1984), thereby demonstrating that creatine synthesis is regulated via GAA production. Furthermore, creatine synthesis has been shown in rats and pigs to be proportional to GAA availability (Da Silva et al. 2009; McBreairty et al. 2013) and human studies have also demonstrated a dose response effect 
of GAA supplementation on the plasma levels of both GAA and creatine (Ostojic et al. 2013). It is possible that variability in the creatine content of the infant diet may also affect the production of GAA, which requires significant amounts of arginine and glycine, which in turn can affect the demand for methyl groups from methionine. Indeed, in the neonatal piglet we have estimated that de novo creatine synthesis consumes $\sim 35 \%$ of dietary methionine and $\sim 20 \%$ of dietary arginine (Brosnan et al. 2009). Expressed relative to protein accretion, this amount of methionine used for creatine synthesis amounts to $30 \%$ of the amount of methionine incorporated into protein, and $10 \%$ for arginine. So this one transmethylation reaction utilizes one-third of dietary methionine in a healthy suckling piglet; if the neonate is fed a low creatine formula, this proportion likely increases dramatically, possibly to the detriment of protein synthesis.

\section{Phosphatidylcholine}

The synthesis of PC via phosphatidylethanolamine methyltransferase (PEMT) involves the sequential transfer of 3 methyl groups to phosphatidylethanolamine (PE) to produce PC (Ridgway and Vance 1987). PC is important for bile and lipoprotein synthesis and quantitatively, the entire hepatic pool of PC is secreted in bile each day (Walkey et al. 1998), highlighting the quantitative significance of PC synthesis. The PEMT pathway is responsible for approximately $30 \%$ of hepatic PC biosynthesis, with the remainder synthesized by the Kennedy pathway via the enzyme CDP-choline:1,2-diacylglycerol cholinephosphotransferase, which transfers phosphocholine to 1,2-diacylglycerol (Li and Vance 2008). While the Kennedy pathway is ubiquitous, PEMT activity is primarily localized to the liver (Cui et al. 1993).

PC can be metabolized to choline and during choline deprivation in rats, choline recycling can occur by increasing PEMT activity via an increase in PE concentration during short term deficiency (Ridgway et al. 1989) or via an upregulation of PEMT expression during 
prolonged deficiency (Cui and Vance 1996). The synthesis of PC via the Kennedy pathway is 3fold higher when PEMT is inhibited demonstrating the coordinated regulation of PC synthesis between the two pathways (Pritchard and Vance 1981). Considering the variability of choline in the infant diet (see below), it is important to understand how dietary choline affects the synthesis of PC via PEMT which in turn affects methyl group availability for other transmethylation reactions (Ilcol et al. 2005).

\section{DNA Methylation}

Another important methyltransferase is DNA methyltransferase (DNMT) which transfers a methyl group to cytosine residues in $\mathrm{CpG}$ dinucleotides of DNA. DNA methylation is an example of an epigenetic alteration, which is a heritable modification of gene expression that does not involve alterations to the DNA sequence (Hanley et al. 2010). These modifications can occur in large regions of DNA, such as repetitive DNA, or can be localized to promoter regions of DNA. Patterns of DNA methylation are established during early development and epigenetic changes near promoter regions of DNA can lead to changes in gene expression (ReamonBuettner et al. 2014; Tate and Bird 1993).

Epigenetic changes to DNA methylation have been implicated in the fetal origins of adult disease hypothesis, which describes how an insult to the fetus can result in permanent negative health effects in later life (McMillen and Robinson 2005). This hypothesis was originally based on epidemiological studies which demonstrated an association between low birth weight and disease in later life (Barker and Osmond 1986; Barker et al. 1989; Gluckman et al. 2008; Godfrey and Barker 2000). This low birth weight or intrauterine growth restriction (IUGR) is mostly due to fetal undernutrition or placental insufficiency, which has been associated with changes to patterns of DNA methylation (Gong et al. 2010; Martínez et al. 2014). It has been 
well established in both human and animal models that IUGR can 'program' an increased risk for hypertension, cardiovascular disease, diabetes and obesity in later life (McMillen and Robinson 2005). Moreover, there is evidence that an imbalance of dietary methionine or methyl nutrients, prenatally or postnatally, play a role in the programming of disease via altered DNA methylation (Kotsopoulos et al. 2008; Langley-Evans 2000; Rees et al. 2006). Although DNA methylation is less quantitatively significant than creatine and $\mathrm{PC}$ synthesis in piglets (McBreairty et al. 2013), the potential for diet to cause epigenetic changes during early development has enormous implications in infant nutrition guidelines.

\section{Remethylation nutrients}

\section{Betaine/Choline}

Choline is highly important during early development and studies have demonstrated a positive association of choline intake with cognitive development (Caudill 2010; McCann et al. 2006; Meck and Williams 2003). The oxidation of choline forms betaine (Kaplan et al. 1993) which functions in the cytosol as both an osmolyte (Häussinger 1996) and as a methyl donor to reform methionine via betaine-homocysteine methyltransferase (BHMT) (Finkelstein et al., 1972), an enzyme primarily active in the liver (Finkelstein 1990; Garrow 1996; McKeever et al. 1991).

The choline concentration in human breast milk is influenced by maternal choline status (Davenport 2015; Ilcol et al. 2005) and is $\sim 1500 \mu \mathrm{M}$ in mature breast milk, consisting of $\sim 35 \%$ from each of glycerophosphocholine and phosphocholine and $\sim 15 \%$ from each of free choline and PC. Moreover, maternal choline supplementation providing approximately twice the choline requirement for adult women leads to $20 \%$ higher levels of total choline in breast milk compared 
to mothers with a choline intake adequate for adult women (Davenport 2015). Infant formula ranges from $700 \mu \mathrm{M}$ choline in soy-derived formula to $1800 \mu \mathrm{M}$ in bovine milk-derived formula and consists mostly of glycerophosphocholine (Holmes-McNary et al. 1996). Free choline in serum is higher in breast fed compared to formula fed infants (Ilcol et al. 2005), highlighting the significance of the variability of choline in the infant diet.

The betaine concentration in human breast milk is only $7 \mu \mathrm{M}$ (Fischer et al. 2010), indicating that the primary source of betaine in the infant is most likely derived from choline which is $\sim 200$-fold higher in breast milk than betaine. In rats, $\sim 85 \%$ of labelled dietary choline and phosphocholine is recovered as betaine in the liver within 24 hours, while only $\sim 50 \%$ and $\sim 15 \%$ of PC and glycerophosphocholine, respectively, are converted to betaine after 24 hours (Cheng et al. 1996), demonstrating the specificity of these choline derivatives as sources of betaine and suggesting that formula-fed infants might not synthesize adequate betaine since most choline in formula occurs as glycerophosphocholine. It is currently unknown whether the variability of choline type and amount in the infant diet leads to variability in the availability of betaine for remethylation in the infant. But inadequate choline and/or betaine intake would likely affect methionine metabolism by enhancing transmethylation via PEMT and limiting remethylation via BHMT.

\section{Folate}

Folate, in the form of 5-methyltetrahydrofolate (5-methyl-THF), can function to provide a methyl group to reform methionine via MS. Although the activity of MS is highest in the liver, the enzyme is ubiquitous with high levels of activity also found in the kidney, brain and pancreas (Brosnan et al. 2009; Finkelstein et al. 1971). Folate is consumed in the diet primarily as a 
polyglutamate derivative and is enzymatically converted via conjugase to an absorbable monoglutamate form, which is further converted to 5-methyl-THF in the intestinal mucosa (Olinger et al. 1973). Using vitamin B12 as a cofactor, 5-methyl-THF provides the methyl group for MS to reform methionine from homocysteine, yielding tetrahydrofolate (THF) (Fujii et al. 1977). Using serine as a methyl source, THF is converted via serine hydroxymethyltransferase to 5,10-methylenetetrahydrofolate (5,10-THF) (Davis et al. 2004; Motokawa and Kikuchi 1971), which can be converted back to 5-methyl-THF via methylenetetrahydrofolate reductase (Kutzbach and Stokstad 1971), completing the folate cycle.

In 1998, the USA and Canada implemented folic acid fortification in a variety of foods including breads and cereals in an attempt to reduce neural tube defects. Although the incidence of neural tube defects has been lowered post-fortification, it has been suggested that fortification could be leading to an excess of folate in the diet (Lucock and Yates 2009; Osterhues et al. 2013). The average total folate concentration in human breast milk is $\sim 80 \mathrm{ng} / \mathrm{mL}$ (Smith et al. 1985 ) and the current global recommendation for the minimum level of folic acid in infant formula is $60 \mathrm{ng} / \mathrm{mL}$ (Koletzko et al. 2005). In addition to dietary sources, folate can be synthesized in the microflora and can provide $\sim 5-10 \%$ of the folate requirement in neonatal piglets (Kim et al. 2004; Asrar and O'Connor 2005). Unlike choline, breast milk folate levels are independent of maternal folate status with no correlation between milk folate concentration and maternal blood folate indexes in women supplemented with folate (Smith et al. 1985). However, deficiency in dietary choline in rats can lead to lower hepatic folate (Selhub et al. 1991) while folate deficiency can lead to depletion of choline in the liver (Kim et al. 1994). This inter-relationship between remethylation nutrients demonstrates how the two remethylation 
pathways compensate for each other highlighting the importance of considering both nutrients when determining dietary requirements in the neonate, when remethylation is in high demand.

\section{Methionine Metabolism Regulation}

\section{Transmethylation and remethylation}

During sulfur amino acid deficiency, protein synthesis is prioritized over transmethylation in the piglet (Bauchart-Thevret et al. 2009); however, less is known about the partitioning of methionine among transmethylation reactions. Studies in adult humans have led to the suggestion that creatine is the primary methyl group consumer (Mudd and Poole 1975), while others have more recently proposed that PC synthesis is quantitatively more significant than creatine due to a 2-3-fold higher SAM utilization by PEMT versus GAMT (Jacobs et al. 2005). Furthermore, experiments in PEMT knockout mice demonstrate $\sim 50 \%$ lower levels of homocysteine after 3 weeks, highlighting the significant demand of PEMT for SAM (Noga and Vance 2003). During early development, methionine is in high demand for both growth and expansion of pools of transmethylation products and the fraction of methionine used for transmethylation reactions is higher in the neonatal versus the 1-month-old piglet (BauchartThevret et al. 2009). This high requirement for transmethylation demonstrates the importance in understanding how variability in the demand for these reactions can affect methionine requirements and availability.

Using $\left[\right.$ methyl $\left.-{ }^{3} \mathrm{H}\right]$ methionine, it has recently been shown that more methionine is used for hepatic PC synthesis compared to creatine synthesis in neonatal piglets (McBreairty et al. 2013). Furthermore, increasing the demand for creatine synthesis via portal infusion of GAA leads to lower methionine availability for incorporation into PC and protein (but no effect on incorporation into DNA) in neonatal piglets (McBreairty et al. 2013). These results were 
reproduced in 3-month-old pigs orally supplemented with GAA for $\sim 3$ weeks (McBreairty et al. 2015). In both models, these data suggest that methionine-derived methyl groups can become limiting even when remethylation nutrients are readily available. These results are concerning due to the variability of creatine in the human infant diet (Edison et al. 2013). Infants receiving cows' milk-based formula receive $36 \%$ of their creatine requirement from the diet while soybased formula provides negligible creatine (Edison et al. 2013), necessitating a much higher creatine synthesis rate. These experimental data strongly suggest that methionine can potentially become limiting in infants fed low creatine diets; studies are needed to quantify the effects of dietary creatine on methionine requirements for PC and protein synthesis in the growing neonate.

The methionine used for protein synthesis and transmethylation reactions comes from both diet and remethylation and interestingly, in piglets it appears that the higher methionine demand for transmethylation reactions in younger neonates is sustained via an increased rate of remethylation (Bauchart-Thevret et al. 2009). Consistent with these developmental changes, human studies have demonstrated that neonates have a higher methionine cycle activity compared to adults (Thomas et al. 2008). Compared to normal weight littermates, IUGR piglets appear to have a reduced capacity to catabolize homocysteine as demonstrated by lower activity levels of both the remethylation enzyme BHMT and the transsulfuration enzyme CGL (MacKay et al. 2012). Furthermore, IUGR piglets have lower methyl incorporation into hepatic PC (McBreairty et al. 2013). It is not known whether these perturbations affect the methionine requirement in the IUGR neonate, but these results highlight the need for a better understanding of the interrelationship between nutrients involved in remethylation and transmethylation reactions, especially considering the quantitative significance of these pathways during early development. 
In vitro studies using rat livers have demonstrated that under standard dietary conditions, both remethylation pathways contribute equally to the reformation of methionine (Finkelstein and Martin 1984). The activity of BHMT is high in the neonatal rat until 3 weeks of age and it declines to a plateau by week 4 (Clow et al. 2008). Due to the maturity of the pig at birth compared to the rat, this result is in keeping with the high rates of remethylation found in the neonatal pig (Bauchart-Thevret et al. 2009) and suggests that remethylation via betaine may be more important in the first weeks of life. Alternatively, developmental changes in MS activity are organ-specific, making it difficult to draw conclusions on the contribution of MS to higher neonatal rates of remethylation (Thompson et al. 2001). Although it has been suggested that under normal dietary conditions, MS is the primary remethylation pathway and BHMT functions in a secondary capacity during times of methionine deficiency (Kim et al. 2005; Park and Garrow 1999) or excess (Finkelstein et al. 1971), most studies are limited to tissue-specific pathways in adult animal models and the quantitative contribution of these individual remethylation pathways to whole body methionine metabolism during early development is not known.

Some studies have focused on homocysteine concentrations as an indirect method to gain insight into the contribution of folate and betaine to whole body remethylation since supplementation with either folate or betaine has the capacity to lower plasma homocysteine (Keser et al. 2013; McRae 2013). BHMT knockout mice fed a standard chow diet have higher levels of plasma homocysteine (Teng et al. 2012) and folate deficient rats also demonstrate elevated levels of homocysteine, highlighting the importance of both BHMT and MS for whole body remethylation (Miller et al. 1994). 
Although these experiments provide valuable insight into methionine metabolism, the regulation of remethylation and transsulfuration is organ-specific (Finkelstein 2007), and the quantification of hepatic enzyme activities and metabolite concentrations do not necessarily represent whole body remethylation metabolism and methionine availability. Although the liver is the primary site for methionine utilization, other organs also contribute significantly to methionine metabolism (Wilson et al. 2009), including the gastrointestinal tract which metabolizes $\sim 20 \%$ of dietary methionine in piglets (Riedijk et al. 2007). Because methionine metabolism is highly variable among tissues, it is important to consider whole body methionine metabolism when determining the relative contributions of folate and betaine to remethylation in the growing neonate. Studies using whole body stable isotope tracers have suggested an equal capacity for folate and betaine to remethylate methionine. Following an intravenous infusion of $\left[{ }^{2} \mathrm{H}_{7}\right]$ methionine in folate- or choline-deficient adult rats, there were no differences in the levels of remethylated methionine in the plasma (Shinohara et al. 2006). Furthermore, a study using the indicator amino acid oxidation method recently determined that both folate and betaine have an equal capacity to reform methionine for protein synthesis when methionine is limiting in the diet of neonatal piglets (McBreairty et al. 2015), suggesting that folate and betaine both have the capacity to affect methionine availability and requirement in the neonate.

The concept of remethylation nutrients sparing methionine is not new (Fukagawa 2006) and cysteine has been shown to spare the methionine requirement by up to $40 \%$ in human adults (Di Buono et al. 2001) and neonatal piglets (Shoveller et al. 2003) demonstrating that the methionine requirement is not static and can be affected by related nutrients. Betaine has been shown to spare methionine in chickens (Dilger et al. 2007); however, experiments in finishing pigs concluded that supplemental betaine cannot replace dietary methionine (Matthews et al. 
2001). Creatine and GAA have also been considered as having potential to spare both methionine and arginine (Dilger et al. 2013); however, supplementation of creatine in 3-monthold pigs for $\sim 3$ weeks did not lead to any changes in homocysteine concentrations or methionine partitioning among protein or PC synthesis or DNA methylation (McBreairty et al. 2015). These differences may be attributed to species-specific renal production of GAA (Edison et al. 2007). Given the emerging evidence that these methionine cycle nutrients can spare methionine, more research is needed to determine if the amount of methionine spared is significant enough to affect methionine requirements, especially during development when demands are more pronounced.

\section{Conclusions}

There is great complexity in determining how the various components of the methionine cycle interact to affect methionine supply and demand. Increasing the demand for the synthesis of one transmethylation product can limit methionine availability for other transmethylation reactions as well as for protein synthesis. Furthermore, dietary supply of nutrients that facilitate remethylation can also affect methionine availability for protein synthesis. The levels of both creatine and choline are particularly variable in the infant diet, with the creatine content of standard infant formula $\sim 3$-fold higher than that of soy-based formula, potentially changing the requirement for endogenous creatine synthesis by 35\% (Edison et al. 2013). Although the choline content of infant diets can also vary, dietary creatine appears to affect methionine availability more significantly, which is concerning considering the high demand for creatine accretion during early development and the capacity for higher creatine synthesis to limit methyl group and methionine availability. Future research should focus on determining whether variation in dietary creatine can affect the dietary methionine requirement in the neonate. 
Furthermore, because the increased rate of transmethylation in the neonate is supported by higher rates of remethylation, the potential for a further increase in the demand for transmethylation to affect requirements for remethylation nutrients should also be considered. This potential effect may be especially important in IUGR infants considering the lower maximum capacity for remethylation and apparent limited methionine availability for PC synthesis in IUGR piglets. It is possible that the IUGR neonate has a lower methionine flux and consequently may be more sensitive changes in demand for creatine synthesis. This review primarily draws on research using the piglet model and although amino acid requirements of the piglet resemble those of the human (Miller and Ullrey 1987), further investigation of the dynamics of methionine metabolism using non-invasive tracer techniques in the human during early development are needed. There is currently a paucity of research on the inter-dependency of nutrients related to methionine metabolism which warrants concern considering their variability in the neonatal diet.

\section{Conflict of interest disclaimer}

The authors declare that there are no conflicts of interest. 


\section{References}

Asrar, F.M. and O'Connor, D.L. 2005. Bacterially synthesized folate and supplemental folic acid are absorbed across the large intestine of piglets. J. Nutr. Biochem. 16(10): 587-93.

Barker, D.J. and Osmond, C. 1986. Infant mortality, childhood nutrition, and ischaemic heart disease in England and Wales. Lancet. 1(8489): 1077-1081.

Barker, D.J. Osmond, C. and Law, C.M. 1989. The intrauterine and early postnatal origins of cardiovascular disease and chronic bronchitis. J. Epidemiol. Community. Health. 43(3): 237-240.

Bauchart-Thevret, C., Stoll, B., Chacko, S. and Burrin, D.G. 2009. Sulfur amino acid deficiency upregulates intestinal methionine cycle activity and suppresses epithelial growth in neonatal pigs. Am. J. Physiol. Endocrinol. Metab. 296(6): 1239-1250.

Bloch, K. and Schoenheimer, R. 1941. The biological precursors of creatine. J. Biol. Chem. 138: 167-194.

Brosnan, J.T., Wijekoon, E.P., Warford-Woolgar, L., Trottier, N.L., Brosnan, M.E., Brunton, J.A. and Bertolo, R.F. 2009. Creatine synthesis is a major metabolic process in neonatal piglets and has important implications for amino acid metabolism and methyl balance. J. Nutr. 139(7): 1292-1297.

Cantoni, G.L. 1951. Activation of methionine for transmethylation. J. Biol. Chem. 189(2): 745754.

Cantoni, G.L. and Vignos, P.J. 1954. Enzymatic mechanism of creatine synthesis. J. Biol. Chem. 209(2): 647-659.

Caudill, M.A. 2010. Pre- and postnatal health: evidence of increased choline needs. J. Am. Diet. Assoc. 110(8): 1198-1206.

Cheng, W.L., Holmes-McNarya, M.Q., Mara, M.H., Lien, E.L. and Zeisel, S.H. 1996.

Bioavailability of choline and choline esters from milk in rat pups. J. Nutr. Biochem. 7(8): $457-$ 464.

Clow, K.A., Treberg, J.R., Brosnan, M.E. and Brosnan, J.T. 2008. Elevated tissue betaine contents in developing rats are due to dietary betaine, not to synthesis. J. Nutr. 138(9): 16411646.

Cui, Z. and Vance, D.E. 1996. Expression of phosphatidylethanolamine N-methyltransferase-2 is markedly enhanced in long term choline-deficient rats. J. Biol. Chem. 271(5): 2839-43.

Cui, Z., Vance, J.E., Chen, M.H., Voelker, D.R. and Vance, D.E. 1993. Cloning and expression of a novel phosphatidylethanolamine N-methyltransferase. A specific biochemical and 
cytological marker for a unique membrane fraction in rat liver. J. Biol. Chem. 268(22): 1665516663.

da Silva, R.P., Nissim, I., Brosnan, M.E. and Brosnan J.T. 2009. Creatine synthesis: hepatic metabolism of guanidinoacetate and creatine in the rat in vitro and in vivo. Am. J. Physiol. Endocrinol. Metab. 296(2): E256-E261.

Davenport, C., Yan, J., Taesuwan, S., Shields, K., West, A.A., Jiang, X., et al. 2015. Choline intakes exceeding recommendations during human lactation improve breast milk choline content by increasing PEMT pathway metabolites. J. Nutr. Biochem. 26(9):903-911.

Davis, S.R., Stacpoole, P.W., Williamson, J., Kick, L.S., Quinlivan, E.P., Coats, B.S., et al. 2004. Tracer-derived total and folate-dependent homocysteine remethylation and synthesis rates in humans indicate that serine is the main one-carbon donor. Am. J. Physiol. Endocrinol. Metab. 286(2): E272-E279.

Di Buono, M., Wykes, L.J., Ball, R.O. and Pencharz, P.B. 2001. Dietary cysteine reduces the methionine requirement in men. Am. J. Clin. Nutr. 74(6):761-766.

Dilger, R.N., Bryant-Angeloni, K, Payne, R.L., Lemme A. and Parsons, C.M. 2013. Dietary guanidino acetic acid is an efficacious replacement for arginine for young chicks. Poult. Sci. 92(1): 171-177.

Dilger, R.N., Garrow, T.A. and Baker, D.H. 2007. Betaine can partially spare choline in chicks but only when added to diets containing a minimal level of choline. J. Nutr. 137(10): 2224-2228.

Edison, E.E., Brosnan, M.E., Aziz, K. and Brosnan, J.T. 2013. Creatine and guanidinoacetate content of human milk and infant formulas: implications for creatine deficiency syndromes and amino acid metabolism. Br. J. Nutr. 110(6): 1075-1078.

Edison, E.E., Brosnan, M.E., Meyer, C. and Brosnan, J.T. 2007. Creatine synthesis: production of guanidinoacetate by the rat and human kidney in vivo. Am. J. Physiol. Renal. Physiol. 293(6): F1799-1F804.

Finkelstein, J.D. 1990. Methionine metabolism in mammals. J. Nutr. Biochem. 1(5): 228-237.

Finkelstein, J.D. 2007. Metabolic regulatory properties of S-adenosylmethionine and Sadenosylhomocysteine. Clin. Chem. Lab. Med. 45(12): 1694-1699.

Finkelstein, J.D. and Chalmers, F.T. 1970. Pyridoxine effects on cystathionine synthase in rat liver. J. Nutr. 100(4): 467-469.

Finkelstein, J.D. and Martin, J.J. 1984. Methionine metabolism in mammals. Distribution of homocysteine between competing pathways. J. Biol. Chem. 259(15): 9508-9513. 
Finkelstein, J.D., Harris, B.J. and Kyle, W.E. 1972. Methionine metabolism in mammals: kinetic study of betaine-homocysteine methyltransferase. Arch. Biochem. Biophys. 153(1): 320-324.

Finkelstein, J.D., Kyle, W. and Harris B.J. 1971. Methionine metabolism in mammals. Regulation of homocysteine methyltransferases in rat tissue. Arch. Biochem. Biophys. 146(1): 84-92.

Fischer, L.M., Da Costa, K.A., Galanko, J., Sha, W., Stephenson, B., Vick, J. and Zeisel, S.H. 2010. Choline intake and genetic polymorphisms influence choline metabolite concentrations in human breast milk and plasma. Am. J. Clin. Nutr. 92(2): 336-346.

Fujii, K., Galivan, J.H. and Huennekens, F.M. 1977. Activation of methionine synthase: further characterization of flavoprotein system. Arch. Biochem. Biophys. 178(2): 662-670.

Fukagawa, N.K. 2006. Sparing of methionine requirements: evaluation of human data takes sulfur amino acids beyond protein. J. Nutr. 136(6 Suppl): 1676S-1681S.

Garrow, T.A. 1996. Purification, kinetic properties, and cDNA cloning of mammalian betainehomocysteine methyltransferase. J. Biol. Chem. 271(37): 22831-22838.

Gluckman, P.D., Hanson, M.A., Cooper, C. and Thornburg, K.L. 2008. Effect of in utero and early-life conditions on adult health and disease. N. Engl. J. Med. 359(1): 1-13.

Godfrey, K.M., and Barker, D.J. 2000. Fetal nutrition and adult disease. Am. J. Clin. Nutr. 71(5 Suppl): 1344S-1352S.

Gong, L., Pan, Y.X. and Chen, H. 2010. Gestational low protein diet in the rat mediates Igf2 gene expression in male offspring via altered hepatic DNA methylation. Epigenetics. 5(7): 619626.

Hanley, B., Dijane, J., Fewtrell, M., Grynberg, A.S., Hummel, R., Junien, C., et al. 2010. Metabolic imprinting, programming and epigenetics - a review of present priorities and future opportunities. Br. J. Nutr. 104(Suppl 1): S1-25.

Huang L, Hogewind-Schoonenboom JE, van Dongen MJ, de Groof F, Voortman GJ, Schierbeek $\mathrm{H}$, et al. 2012. Methionine requirement of the enterally fed term infant in the first month of life in the presence of cysteine. Am. J. Clin. Nutr. 95(5): 1048-1054.

Häussinger, D. 1996. The role of cellular hydration in the regulation of cell function. Biochem. J. 313(Pt 3): 697-710.

Holmes-McNary, M.Q., Cheng, W.L., Mar, M.H., Fussell, S. and Zeisel, S.H. 1996. Choline and choline esters in human and rat milk and in infant formulas. Am. J. Clin. Nutr. 64(4): 572-576. 
Ilcol, Y.O., Ozbek, R., Hamurtekin, E. and Ulus, I.H. 2005. Choline status in newborns, infants, children, breast-feeding women, breast-fed infants and human breast milk. J. Nutr. Biochem. 16(8): 489-499.

Jacobs, R.L., Stead, L.M., Devlin, C., Tabas, I., Brosnan, M.E., Brosnan, J.T. and Vance, D.E. 2005. Physiological regulation of phospholipid methylation alters plasma homocysteine in mice. J Biol Chem. 280(31): 28299-28305.

Kaplan, C.P., Porter, R.K., Br, M.D. and Brand, M.D. 1993. The choline transporter is the major site of control of choline oxidation in isolated rat liver mitochondria. FEBS. Lett. 321(1): 24-26.

Keser, I., Ilich, J.Z., Vrkić, N., Giljević, Z. and Colić Barić, I. 2013. Folic acid and vitamin B(12) supplementation lowers plasma homocysteine but has no effect on serum bone turnover markers in elderly women: a randomized, double-blind, placebo-controlled trial. Nutr. Res. 12(1): 20-25.

Kim Y.I. 2005. Nutritional epigenetics: impact of folate deficiency on DNA methylation and colon cancer susceptibility. J. Nutr. 135(11): 2703-2709.

Kim, T.H., Yang, J., Darling, P.B. and O'Connor, D.L. 2004. A large pool of available folate exists in the large intestine of human infants and piglets. J. Nutr. 134(6): 1389-1394.

Kim, Y.I., Miller, J.W., da Costa, K.A., Nadeau, M., Smith, D., Selhub, J., et al. 1994. Severe folate deficiency causes secondary depletion of choline and phosphocholine in rat liver. J. Nutr. 124(11): 2197-2203.

Koletzko, B., Baker, S., Cleghorn, G., Neto, U.F., Gopalan, S., Hernell, O., et al. 2005. Global standard for the composition of infant formula: recommendations of an ESPGHAN coordinated international expert group. J. Pediatr. Gastroenterol. Nutr. 41(5): 584-599.

Kotsopoulos, J., Sohn, K.J. and Kim, Y.I. 2008. Postweaning dietary folate deficiency provided through childhood to puberty permanently increases genomic DNA methylation in adult rat liver. J. Nutr. 138(4): 703-709.

Kutzbach, C. and Stokstad, E.L. 1971. Mammalian methylenetetrahydrofolate reductase. Partial purification, properties, and inhibition by S-adenosylmethionine. Biochim. Biophys. Acta. 250(3): 459-477.

Langley-Evans, S.C. 2000. Critical differences between two low protein diet protocols in the programming of hypertension in the rat. Int. J. Food. Sci. Nutr. 51(1): 11-17.

Li, Z. and Vance, D.E. 2008. Phosphatidylcholine and choline homeostasis. J. Lipid. Res. 49(6): 1187-1194.

Lucock, M. and Yates, Z. 2009. Folic acid fortification: a double-edged sword. Curr. Opin. Clin. Nutr. Metab. Care. 12(6): 555-564. 
MacKay, D.S., Brophy, J.D., McBreairty, L.E., McGowan, R.A. and Bertolo, R.F. 2012. Intrauterine growth restriction leads to changes in sulfur amino acid metabolism, but not global DNA methylation, in Yucatan miniature piglets. J. Nutr. Biochem. 23(9): 1121-1127.

Martínez, D., Pentinat, T., Ribó, S., Daviaud, C., Bloks, V.W., Cebrià., et al. 2014. In utero undernutrition in male mice programs liver lipid metabolism in the second-generation offspring involving altered Lxra DNA methylation. Cell. Metab. 19(6): 941-951.

Matthews, J.O., Southern, L.L., Higbie, A.D., Persica, M.A. and Bidner, T.D. 2001. Effects of betaine on growth, carcass characteristics, pork quality, and plasma metabolites of finishing pigs. J. Anim. Sci. 79(3): 722-728.

McBreairty, L.E., Robinson, J.L., Harding, S.V., Randell, E.W., Brunton, J.A. and Bertolo, R.F. 2015. Betaine is as effective as folate at re-synthesizing methionine for protein synthesis during moderate methionine deficiency in piglets. Eur. J. Nutr. Epub ahead of print.

McBreairty, L.E., Robinson, J.L., Furlong, K.R., Brunton, J.A. and Bertolo, R.F. 2015. Guanidinoacetate is more effective than creatine at enhancing tissue creatine stores while consequently limiting methionine availability in Yucatan miniature pigs. PLoS. One. 10(6).

McBreairty, L.E., McGowan, R.A., Brunton, J.A. and Bertolo, R.F. 2013. Partitioning of [methyl-3H]methionine to methylated products and protein is altered during high methyl demand conditions in young Yucatan miniature pigs. J. Nutr. 143(6): 804-809.

McCann J.C., Hudes M. and Ames B.N. 2006. An overview of evidence for a causal relationship between dietary availability of choline during development and cognitive function in offspring. Neurosci. Biobehav. Rev. 30(5): 696-712.

McGuire, D.M., Gross, M.D., van Pilsum, J.F. and Towle, H.C. 1984. Repression of rat kidney L-arginine:glycine amidinotransferase synthesis by creatine at a pretranslational level. J. Biol. Chem. 259(19): 12034-12038.

McKeever, M.P., Weir, D.G., Molloy, A. and Scott, J.M. 1991. Betaine-homocysteine methyltransferase: organ distribution in man, pig and rat and subcellular distribution in the rat. Clin. Sci. (Lond). 81(4): 551-556.

McMillen, I.C. and Robinson, J.S. 2005. Developmental origins of the metabolic syndrome: prediction, plasticity, and programming. Physiol. Rev. 85(2): 571-633.

McRae, M.P. 2013. Betaine supplementation decreases plasma homocysteine in healthy adult participants: a meta-analysis. J. Chiropr. Med. 12(1): 20-25.

Meck, W.H. and Williams, C.L. 2003. Metabolic imprinting of choline by its availability during gestation: implications for memory and attentional processing across the lifespan. Neurosci.

Biobehav. Rev. 27(4): 385-399. 
Miller, J., Nadeau, M., Smith, J., Smith, D. and Selhub, J. 1994. Folate-deficiency-induced homocysteinaemia in rats: disruption of S-adenosylmethionine's co-ordinate regulation of homocysteine metabolism. Biochem. J. 298(Pt 2): 415-419.

Millian, N.S. and Garrow T.A. 1998. Human betaine-homocysteine methyltransferase is a zinc metalloenzyme. Arch. Biochem. Biophys. 356(1): 93-98.

Miller, E.R. and Ullrey, D.E. 1987. The pig as a model for human nutrition. Annu. Rev. Nutr. 7: 361-382.

Motokawa, Y. and Kikuchi, G. 1971. Glycine metabolism in rat liver mitochondria. V. Intramitochondrial localization of the reversible glycine cleavage system and serine hydroxymethyltransferase. Arch. Biochem. Biophys. 146(2): 461-464.

Mudd, S.H. and Poole, J.R. 1975. Labile methyl balances for normal humans on various dietary regimens. Metabolism. 24(6): 721-735.

Noga, A.A. and Vance, D.E. 2003. Insights into the requirement of phosphatidylcholine synthesis for liver function in mice. J. Lipid. Res. 44(10): 1998-2005.

Olinger, E.J., Bertino, J.R. and Binder, H.J. 1973. Intestinal Folate Absorption. J. Clin. Investig. 52(9): 2138-2145.

Osterhues, A., Ali, N.S. and Michels, K.B. 2013. The role of folic acid fortification in neural tube defects: a review. Crit. Rev. Food. Sci. Nutr. 53(11): 1180-1190.

Ostojic, S.M., Niess, B., Stojanovic, M. and Obrenovic, M. 2013. Creatine metabolism and safety profiles after six-week oral guanidinoacetic acid administration in healthy humans. Int. J. Med. Sci. 10(2): 141-147.

Park, E.I. and Garrow, T.A. 1999. Interaction between dietary methionine and methyl donor intake on rat liver betaine-homocysteine methyltransferase gene expression and organization of the human gene. J. Biol. Chem. 274(12): 7816-7824.

Pritchard, P.H. and Vance, D.E. 1981. Choline metabolism and phosphatidylcholine biosynthesis in cultured rat hepatocytes. Biochem. J. 196(1): 261-267.

Reamon-Buettner, S.M., Buschmann, J. and Lewin, G. 2014. Identifying placental epigenetic alterations in an intrauterine growth restriction (IUGR) rat model induced by gestational protein deficiency. Reprod. Toxicol. 45: 117-124.

Rees, W.D., Hay, S.M. and Cruickshank, M. 2006. An imbalance in the methionine content of the maternal diet reduces postnatal growth in the rat. Metabolism. 55(6): 763-770. 
Ridgway, N.D. and Vance, D.E. 1987. Purification of phosphatidylethanolamine Nmethyltransferase from rat liver. J. Biol. Chem. 262(35): 17231-9.

Ridgway, N.D., Yao, Z. and Vance, D.E. 1989. Phosphatidylethanolamine levels and regulation of phosphatidylethanolamine N-methyltransferase. J. Biol. Chem. 264(2): 1203-1207.

Riedijk, M.A., Stoll, B., Chacko, S., Schierbeek, H., Sunehag, A.L., van Goudoever, J.B. and Burrin, D.G. 2007. Methionine transmethylation and transsulfuration in the piglet gastrointestinal tract. Proc. Natl. Acad. Sci. USA. 104(9): 3408-3413.

Schubert, H.L., Blumenthal, R.M. and Cheng, X. 2003. Many paths to methyltransfer: a chronicle of convergence. Trends. Biochem. Sci. 28(6): 329-335.

Selhub, J., Seyoum, E., Pomfret, E.A. and Zeisel, S.H. 1991. Effects of choline deficiency and methotrexate treatment upon liver folate content and distribution. Cancer. Res. 51(1): 16-21.

Shinohara, Y., Hasegawa, H., Ogawa, K., Tagoku, K. and Hashimoto, T. 2006. Distinct effects of folate and choline deficiency on plasma kinetics of methionine and homocysteine in rats. Metabolism. 55(7): 899-906.

Shoveller, A.K., Brunton, J.A., House, J.D., Pencharz, P.B. and Ball, R.O. 2003. Dietary cysteine reduces the methionine requirement by an equal proportion in both parenterally and enterally fed piglets. J. Nutr. 133(12): 4215-4224.

Smith, A.M., Picciano, M.F. and Deering, R. H. 1985. Folate intake and blood concentrations of term infants. Am. J. Clin. Nutr. 41(3): 590-598.

Tate, P.H. and Bird, A.P. 1993. Effects of DNA methylation on DNA-binding proteins and gene expression. Curr. Opin. Genet. Dev. 3(2): 226-231.

Teng, Y., Cerdena, I. and Zeisel, S.H. 2012. Homocysteinemia in mice with genetic betaine homocysteine S-methyltransferase deficiency is independent of dietary folate intake. J. Nutr. 142(11): 1964-1967.

Thomas, B., Gruca, L.L., Bennett, C., Parimi, P.S., Hanson, R.W. and Kalhan, S.C. 2008. Metabolism of methionine in the newborn infant: response to the parenteral and enteral administration of nutrients. Pediatr. Res. 64(4): 381-386.

Thompson, H.R., Jones, G.M. and Narkewicz, M.R. (2001). "Ontogeny of hepatic enzymes involved in serine- and folate-dependent one-carbon metabolism in rabbits.” Am. J. Physiol. Gastrointest. Liver. Physiol. 280(5): G873-G878.

Walker, J.B. 1960. Metabolic control of creatine biosynthesis. I. Effect of dietary creatine. J. Biol. Chem. 235: 2357-2361. 
Walkey, C.J., Yu, L., Agellon, L.B. and Vance, D.E. 1998. Biochemical and evolutionary significance of phospholipid methylation. J. Biol. Chem. 273(42): 27043-27046.

Wilson, F.A., van den Borne, J.J., Calder, A.G., O'Kennedy, N., Holtrop, G., Rees, W.D. and Lobley, G.E. 2009. Tissue methionine cycle activity and homocysteine metabolism in female rats: impact of dietary methionine and folate plus choline. Am. J. Physiol. Endocrinol. Metab. 296(4): E702-E713.

Zlotkin, S.H. and Anderson, G.H. 1982. The development of cystathionase activity during the first year of life. Pediatr. Res. 16(1): 65-68. 
Fig. 1. Methionine metabolism overview

5- $\mathrm{CH}_{3}$-THF, 5-Methyltetrahydrofolate; 5,10- $\mathrm{CH}_{2}$-THF, 5,10-Methylenetetrahydrofolate; BHMT, Betaine-homocysteine methyltransferase; CBS, Cystathionine $\beta$-synthase; CGL, Cystathionine $\gamma$ lyase; DMG, Dimethylglycine; DNMT, DNA methyltransferase; GAA, Guanidinoacetate; GAMT, Guanidinoacetate methyltransferase; MAT, Methionine adenosyltransferase; MS, Methionine synthase; MTHFR, Methylenetetrahydrofolate reductase; PE, Phosphatidylethanolamine; PC, Phosphatidylcholine; PEMT, Phosphatidylethanolamine $N$ methyltransferase; SAHH, $S$-adenosylhomocysteine hydrolase; THF, tetrahydrofolate. 


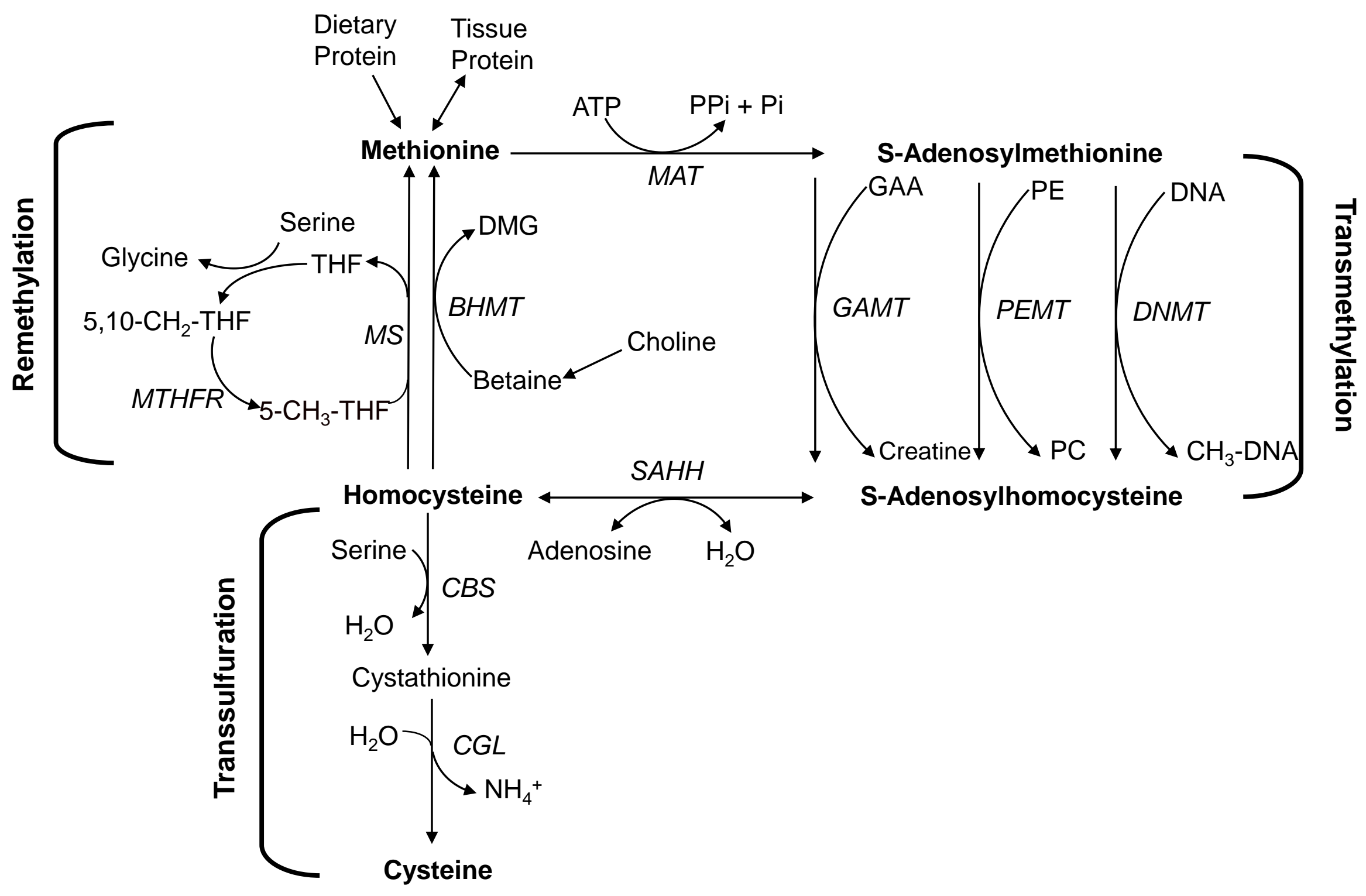

